\title{
Kinetic Modeling of the Electron Current Collection to a Moving Bare Electrodynamic Tether
}

\author{
Éric Choinière and Brian E. Gilchrist \\ University of Michigan, Ann Arbor, Michigan 48109 \\ (734) 763-5357, echoinie@umich.edu
}

\begin{abstract}
Tether electron current collection in the Orbital Motion Limited regime is the key mechanism allowing for power and/or thrust generation applications of space electrodynamic tethers. This paper presents a new approach, based on a kinetic model, for the accurate analysis of the electron collection problem to a bare tether moving in a collisionless plasma. The drift velocity associated with the translational speed of the tether is incompatible with 1-D, cylindrically symmetric models (Laframboise, 1966; Sanmartín and Estes, 1999) and its effects on current collection are not well understood. A kinetic model is developed for the two-dimensional plasma surrounding the tether. It consists in solving, self-consistently, the Vlasov and Poisson equations through a semi-analytical, semi-numerical process. A Maxwellian velocity distribution is assumed for the plasma species (electrons and ions) at the outer boundary of the solution space; no assumption is made regarding the velocity distributions in the vicinity of the tether. Initial results are validated with Langmuir cylindrical probe theory in the ion and electron saturation regimes as well as the electron retardation regime. Work is underway to allow the use of the model for cases with a drifting plasma.
\end{abstract}

\section{INTRODUCTION}

Bare electrodynamic space tethers are under consideration for applications such as power generation and propellantless propulsion for orbiting spacecraft. In these applications, one of the primary concerns is the ability of the system to collect electrons from the surrounding ionospheric plasma in order to maximize the amount of electrical power or thrust provided. This value increases with the magnitude of the current flowing on the tether, which in turn is, in part, limited by the tether's ability to collect electrons from the surrounding plasma.

Analytical models are available (Laframboise, 1966; Sanmartín and Estes, 1999) for the electron current collection problem in the limiting case of the Orbital Motion Limit regime and a stationary plasma with respect to the probe. A numerical model was also developed (Laframboise, 1966) for the general case which was based on a self-consistent, 1-D solution of Vlasov and Poisson's equations for a cylindrically symmetric structure and boundary conditions. This model did not restrict its application to the OML regime, however it did not allow the inclusion of a plasma drift or modeling of probes with arbitrary geometries.

This paper presents a novel approach, based on a poly-energetic kinetic model, for the accurate analysis of the electron/ion current collection problem to a bare tether in a collisionless, drifting plasma. This technique is a 2-D extension of the self-consistent 1-D numerical model shown in (Laframboise, 1966), and allows inclusion of a drift velocity to the background plasma as well as opening the possibility for accurate modeling of tethers of arbitrary cross-sections.

We will first provide a physical and mathematical description of the problem under consideration. The proposed iterative resolution

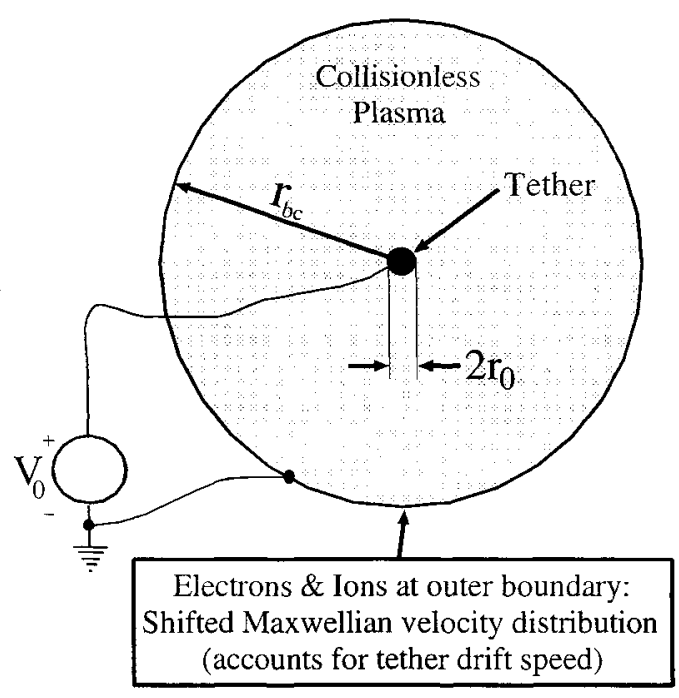

FIGURE 1. Basic Geometry of the Artificial Tether Problem. 
scheme will then be introduced, together with the associated computational issues. Finally, we will show the computation results that were obtained in the initial validation of the proposed algorithm and compare them with known analytical results. Although cases featuring a drifting plasma are supported by the theory presented here, work is underway to obtain results for such cases.

\section{DESCRIPTION OF THE 2-D CONDUCTING TETHER PROBLEM}

Figure 1 presents the basic geometry being considered here. The tether is assumed to be a cylinder of infinite length, surrounded by a plasma comprised of electrons and ions. In the simulation, an outer equipotential shell is placed at a radius $r_{b c}$ from the center of the tether, in order to simulate the background plasma potential and limit the computational region. A potential $V_{0}$ with respect to the plasma potential is specified at the probe surface. Although no assumptions are made concerning the velocity distributions of the plasma species within the computational region, it is assumed that both the ion and electron populations have a drifting Maxwellian velocity distribution at the outer shell, that is, in the background plasma:

$$
f_{e, i}\left(v_{x_{2}} v_{y}\right)=\frac{n_{0} m_{e, i}}{2 \pi e T_{e, i}} \exp \left\{-\frac{m_{e, i}}{2 e T_{e, i}}\left(\left(v_{x}-v_{d}\right)^{2}+1 v_{y}^{2}\right)\right\}
$$

In order to simplify the initial development of the modeling technique, we assume a collisionless, unmagnetized quiescent plasma. Additionally, it is assumed that the electric field vanished for $r>r_{b c}$ and that the potential distribution is piecewise bilinear. In other words, it varies bilinearly within any of the triangles of the mesh.

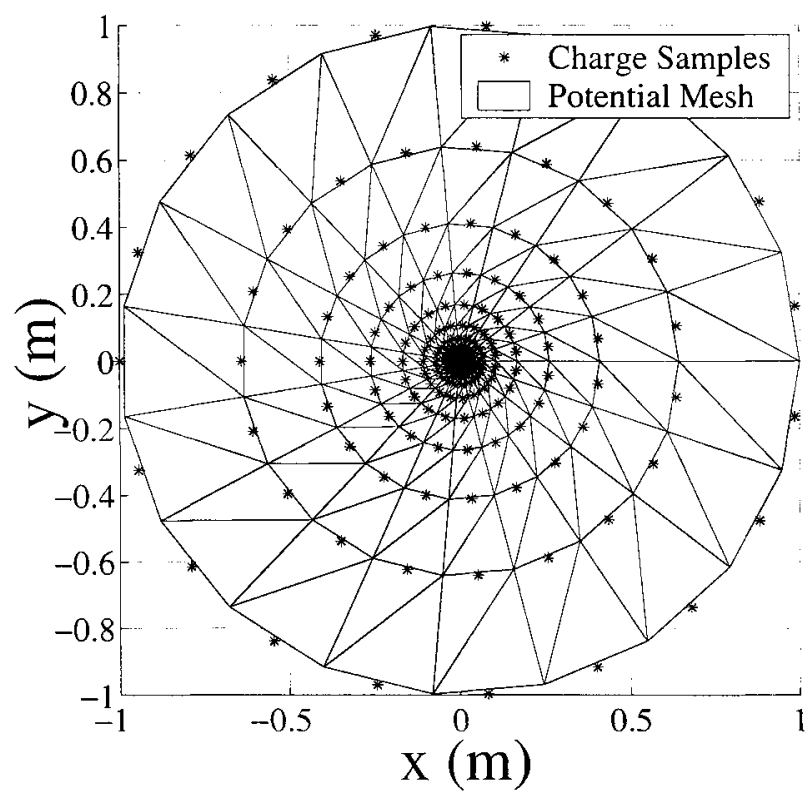

FIGURE 2. Triangulation Schemes for the Potential and Charge Distributions.

As the last assumption specifies, the potential and charge density distributions are sampled on two separate fixed grids of points, which together form a set of adjacent triangles. Figure 2 illustrates the triangulation schemes for both the potential and charge density distributions. The meshes are spaced logarithmically along the radial direction, which is justified by the expected large variations near the tether as opposed to the smoother variations further out. The consequence of the bilinearity assumption for the potential is that both components of the electric field, $E_{x}$ and $E_{y}$, will be piecewise constant (constant on any single triangle).

\section{ITERATIVE CONSISTENT 2-D PLASMA SOLVER}

In order to obtain a consistent solution for the electric fields and the density distributions for both the ion and electron species, we need to solve, self-consistently, Vlasov's equation for each species and Poisson's equation for the electric potential and charges, while satisfying the above-mentioned boundary conditions.

An iterative scheme was developed using both a Vlasov solver and a Poisson solver. In the following we will describe both solvers and present the iterative scheme that was used.

\section{Vlasov solver}

For a collisionless species in a quiescent, unmagnetized plasma, Vlasov's equation in 2-D can be written as:

$$
\frac{d f}{d t}-v_{x} \frac{\partial f_{e, i}}{\partial x}+v_{y} \frac{\partial f_{e, i}}{\partial y}+\frac{q_{e, i} E_{x}(x, y)}{m_{e, i}} \frac{\partial f_{e, i}}{\partial v_{x}}+\frac{q_{e, i} E_{y}(x, y)}{m_{e, i}} \frac{\partial f_{e, i}}{\partial v_{y}}=0
$$

Given a known electric field distribution $\vec{E}(x, y)$ and the outer "drifting Maxwellian" boundary condition for the velocity distributions, equation (2) can be used to solve for the velocity distributions $f_{e, i}\left(x, y, v_{x}, v_{y}\right)$ of both the electrons 
and ions. The species densities are then obtained by integration over all velocity space:

$$
n_{e, i} \sqsupset \iint f_{e, i}\left(x, y, v_{x}, v_{y}\right) d v_{x} d v_{y}
$$

and the total charge density results from $\rho\{x, y)=q_{i} n_{i}-e n_{e}$.

Equation (2) specifies that the distribution function $f_{e, i}\left(x, y, v_{x}, v_{y}\right)$ is constant along particle orbits in a given electric field distribution. Using the specified boundary condition for the velocity distribution at the outer shell, the value for $f_{e, i}\left(x, y, v_{x}, v_{y}\right)$ for any point and velocity located on a trajectory originating from the outer shell can be inferred. This can be done by tracking the particle's trajectory back in time until it hits the outer boundary. Any trajectory not originating from the outer shell is deemed unpopulated (Laframboise, 1966). Such is the case for those trajectories originating from the tether itself, which does not emit charged particles, as well as trapped trajectories which have no sources of particles in the collisionless case.

Figure 3 illustrates the particle tracking process. The trajectories are tracked analytically from one edge of the mesh to another, assuming a constant electric field within any given triangle of the mesh. Every subtrajectory is thus resolved by computing the intersection of a quadratic parametric curve with a segment on the mesh. This technique is much more efficient than using a fixed time step particle pusher, since the amount of computations necessary for one trajectory depends on the number of edges being crossed rather than the number of time steps necessary to reach a boundary. Also, given the assumption of a piecewise-bilinear potential distribution, it provides exact (nearly exact if we account for roundoff errors) conservation of energy along orbits, which contributes to the accuracy of the overall approach.

To obtain a value for the particle density at a given point, one needs to integrate the values obtained for $f_{e, i}\left(x, y, v_{x}, v_{y}\right)$ using the integral shown in equation (3). To limit the computational task, the domain of integration is restricted to a limited region outside of which the

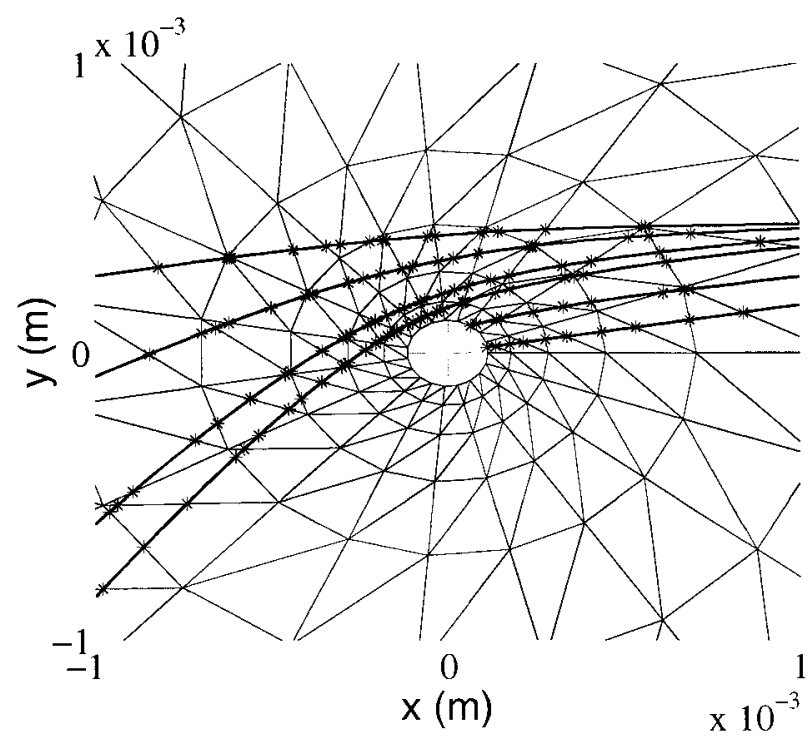

FIGURE 3. Example of the Semi-analytical Particle Tracking Process Through the Potential Mesh.

velocity distribution function is known to be very low. Knowing that the velocity distribution function anywhere on the outer boundary is given by equation (1), we can use conservation of energy to restrict the integration domain to a certain range of kinetic energies. At the outer boundary, a range of integration $K_{\max }=\kappa T_{e, i}$ (units of eV's) is specified in terms of the species temperature (typically, $K=10$ ). At this location, the integral could be performed accurately over a disk of radius:

$$
\Delta v=\sqrt{\frac{2 e K_{\max }}{m_{e, i}}} \neg \sqrt{\frac{2 e \kappa T_{e, i}}{m_{e, i}}}
$$

centered around the center of the Maxwellian distribution, located at $v_{x}=v_{d}, v_{y}=0$. This disk is located between 2 rings of velocity magnitudes $\left|v_{\min }\right|$ and $\left|v_{\max }\right|$ given by:

$$
v_{\min }=\max \left(0, v_{d}-\Delta v\right) \quad v_{\max }-v_{d} H \Delta v
$$

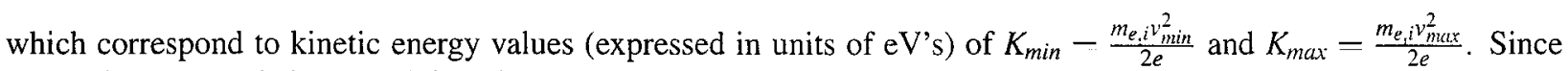
energy is conserved along particle trajectories, we can infer the following limits of integration along the kinetic energy axis at a location situated at a potential $V$ (in volts):

$$
\begin{gathered}
K_{\min }=\max \left(0, \frac{m_{e, i} v_{\min }^{2}}{2 e}-\frac{q_{e, i}}{e} V\right) \\
K_{\max }=\max \left(0, \frac{m_{e, i} v_{\max }^{2}}{2 e}-\frac{q_{e, i}}{e} V\right)
\end{gathered}
$$




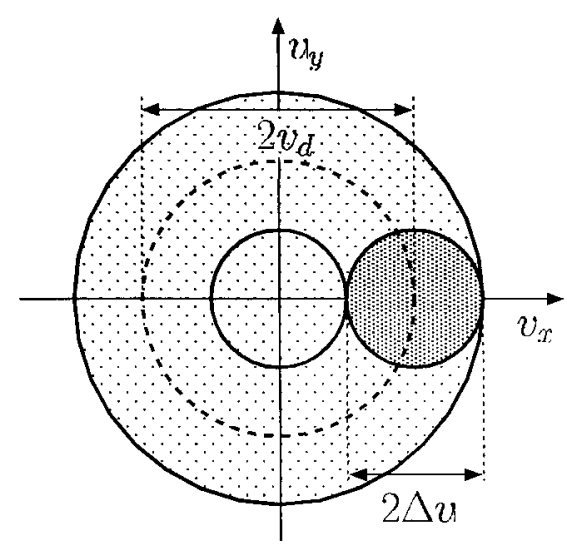

(a)

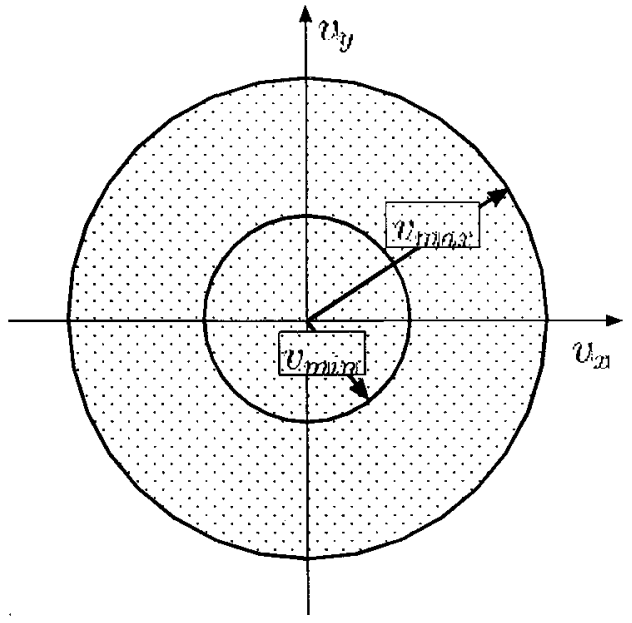

(b)

FIGURE 4. (a) Domain of Integration of the Velocity Distribution Function at the Outer Boundary $(V=0)$. The shaded region corresponds to the bulk of the drifting Maxwellian population. (b) Domain of Integration of the Velocity Distribution Function at any Given Point in Space.

which can be expressed in terms of velocity magnitudes:

$$
v_{\min }=\sqrt{\frac{2 e K_{\min }}{m_{e, i}}} \quad v_{\max }=\sqrt{\frac{2 e K_{\max }}{m_{e, i}}}
$$

Figure 4 shows the corresponding velocity domains of integration. The 2-D integration is performed in cylindrical coordinates, using 2 embedded 7-point adaptive Newton-Cotes quadrature rules (the integration routine "DQNC79" was used from the SLATEC Common Mathematical Library, distributed by Netlib at www.netlib.org). The dynamic integration routine is performed at all nodes on the charge density mesh.

This process described above constitutes the "Vlasov solver" which computes the charge density distribution from a known potential distribution. It accounts for the largest part of the computational complexity of the technique presented here.

\section{Poisson solver}

The Poisson solver is a routine which computes the potential distribution produced by the sum of a given plasma charge density distribution and the surface charge distributions which are required to obtain an equipotential outer boundary $\left(E_{\theta}-0\right)$ and meet the potential boundary condition $\left(V-V_{0}\right.$ at the probe surface). Surface charges are located on the probe surface as well as on the surface of the outer boundary.

As a first step, Poisson's equation and the outer boundary condition:

$$
\varepsilon_{0} \nabla^{2} V(x: y)=\left.\rho(\mid x, y)_{1} \quad V\right|_{r=r_{b c}}-0 \text { Volts }
$$

can be solved for to obtain the "plasma contribution" to the complete potential distribution:

$$
V_{\text {plasma }}\left([r, \theta)-\frac{-1}{4 \pi \varepsilon_{0}} \iint r^{\prime} d r^{\prime} d \theta^{\prime} \times \rho\left(r^{\prime}, \theta^{\prime}\right) \ln \left(\frac{r^{2}+r^{\prime 2}-2 r^{\prime} r \cos \left(\theta-\theta^{\prime}\right)}{r_{b c}^{2}+r^{\prime 2}-2 r^{\prime} r_{b c} \cos \left(\theta-\theta^{\prime}\right)}\right)\right.
$$

where we find the logarithmic dependence characteristic of the infinite cylinders of charge represented in a 2D problem (Cheng, 1989). A point-matching technique is then used in order to find the required surface charge distributions on the probe and the outer boundary that will result in a total potential $V_{0}$ at all nodes located on the surface of the 
probe, as well as a vanishing total tangential electric field $E_{\theta} \neg 0$ at the outer boundary. The "probe surface charge" and "outer boundary surface charge" contributions to the complete plasma distribution are, respectively:

$$
V_{p s c}(r, \theta)-\frac{-r_{0}}{4 \pi \varepsilon_{0}} \iint d r^{\prime} d \theta^{\prime} \times \rho_{s}\left(r_{0}, \theta^{\prime}\right) \ln \left(\frac{r^{2}+1 r_{0}^{2}-2 r_{0} r \cos \left(\theta-\theta^{\prime}\right)}{r_{b c}^{2}+r_{0}^{2}-2 r_{0} r_{b c} \cos \left(\theta-\theta^{\prime}\right)}\right)
$$

and

$$
V_{o b s c}(r, \theta)=\frac{-r_{b c}}{4 \pi \varepsilon_{0}} \iint d r^{\prime} d \theta^{\prime} \times \rho_{s}\left(r_{b c}, \theta^{\prime}\right) \ln \left(\frac{r^{2}+r_{b c}^{2}-2 r_{b c} r \cos \left(\theta-\theta^{\prime}\right)}{r_{b c}^{2}+r_{b c}^{2}-2 r_{b c}^{2} \cos \left(\theta-\theta^{\prime}\right)}\right)
$$

where $\rho_{s}\left(r_{0}, \theta\right)$ and $\rho_{s}\left(r_{b c}, \theta\right)$ are obtained using a point-matching technique (Stutzman and Thiele, 1981) that enforces $V_{p s c}\left(r_{0}, \theta\right)+V_{o b s c}\left(r_{0}, \theta\right)=V_{0}-V_{\text {plasma }}\left(r_{0}, \theta\right)$ at all nodes on the probe surface as well as a vanishing total tangential electric field $E_{\theta}-0$ at the outer boundary.

The total potential distribution is obtained from the sum of equations (10), (11) and (12). Since the integrals are performed over piecewise bilinear functions, the complete Poisson solver (including the point-matching operation) can be written in the form of a linear matrix operator, which makes it computationally trivial compared to the Vlasov solver.

\section{Iterative Resolution Technique}

Using the Poisson and Vlasov solvers, we would like to find a solution for the potential and charge distributions which simultaneously satisfies the Poisson and Vlasov equations. Figure 5 depicts the general fixed-point operator composed of both the Poisson and Vlasov solvers. The fixedpoint operator takes a potential distribution $\vec{V}$ at its input and generates a new estimate $\vec{V}^{\prime}$ :

$$
\vec{V}^{\prime}=\overrightarrow{\mathbf{g}}(\vec{V})
$$

There are known difficulties arising in solving such a problem (Laframboise, 1966). Simple iteration of the fixed point operator does not in general yield convergence, since it is a non-contractive mapping (Sikorski, 2001). Instead, we have used the Newton method which necessitates the Jacobian matrix of the fixed point operator and is equivalent to iterating the following fixed point function:

$$
\vec{V}^{\prime}-\vec{h}(\vec{V})-\vec{V}+i\left(\mathbf{J}_{\mathbf{g}}(\vec{V})-\mathbf{I}\right)^{-1}(\vec{V}-\overrightarrow{\mathbf{g}}(\vec{V}))
$$

where $\mathbf{J}_{\mathbf{g}}(\overrightarrow{\mathbf{V}})$ is the Jacobian matrix of operator $\overrightarrow{\mathbf{g}}(\cdot)$ evaluated at $\vec{V}$. If there are $N$ unknowns ( $N$ potential nodes), the Jacobian matrix is a $N \times N$ matrix and is defined by:

$$
\mathbf{J}_{\mathbf{g}}(i, j)-\frac{\partial g_{i}}{\partial V_{j}}, \quad i=1, \ldots, N, \quad j=1, \ldots, N .
$$

Solving equation (14) is equivalent to finding the solution to the linearized

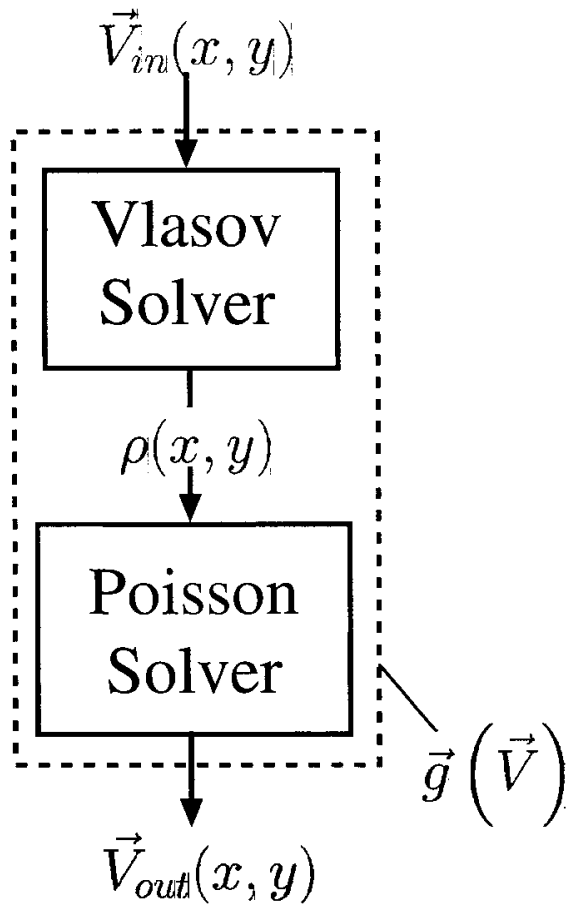

FIGURE 5. Fixed Point Operator Comprised of Both the Poisson and Vlasov Solvers. operator $\overrightarrow{\mathbf{g}}$ near an operating point $\vec{V}$. Successive linearizations should lead to the solution to the nonlinear problem. Using finite differences to compute an approximation for $\mathbf{J}_{\mathbf{g}}$ would be prohibitively expensive computationally. Instead, it was decided to implement the computation of $\mathbf{J}_{\mathbf{g}}$ directly. This involves combining the $\mathbf{J} a c o b i a n s$ of every sample of the velocity distribution function that was taken at that particular iteration using all the operators that were applied to those sample results. To obtain the Jacobians of velocity distribution samples, the analytical Jacobians obtained at the outer boundary using equation (1) have to be propagated and transformed along each particle trajectory.

\section{Software Implementation}

The iterative resolution high-level algorithm and the Poisson solver, both fairly light computationally, were implemented in Matlab. The Vlasov solver, being much heavier computationally, was implemented in Fortran 90 using a 
parallel processing scheme based on the Parallel Virtual Machine library (www.epm.ornl.gov/pvm/pvm_home.html). 20 to 50 workstation nodes were used concurrently to form the Vlasov solver. Simulation times were on the order of 15-30 minutes for the results presented in the following section.

\section{SIMULATION RESULTS \& VALIDATION}

In order to gain confidence in this modeling tool, some validation runs were performed with simple cases that can be checked against known theories. Figure 6 presents the results obtained for the I-V curve of a Langmuir cylindrical probe of radius $r_{0}=$ $0.14 \mathrm{~mm}$ in a Xenon plasma $\left(m_{i}-2.18 \times 10^{-25} \mathrm{~kg}\right)$ with a density $n_{0}-1.42 \times 10^{16} \mathrm{~m}^{-3}$ and a temperature of $T_{e}-T_{i}=1.07 \mathrm{eV}$. The I-V curve has been split in two separate parts to emphasize the small variation in the ion saturation region.

In both the ion and electron saturation regions, our results are in good agreement with the Orbit Motion Limit theory, which is valid in the present case for large voltages due to the fact that the probe radius is fairly small in terms of the Debye length $\left(\lambda_{d}-0.062 \mathrm{~mm}\right)$. The OML expression used for comparison is (Sanmartín and Estes, 1999):

$$
I_{o m l}=2 r_{0} e n_{0} \sqrt{\frac{2 e|V|}{m_{e: i}}}
$$

where $m_{e}$ and $m_{i}$ were used, respectively, in the electron and ion saturation regions.

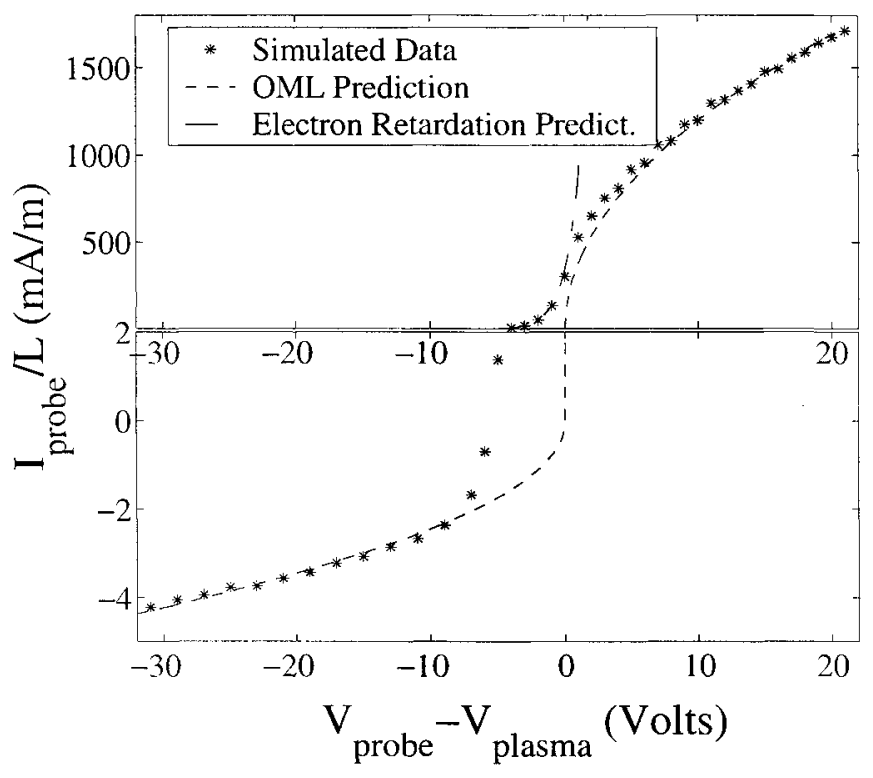

FIGURE 6. I-V Characteristic Curve of a Langmuir Probe. Note that the ion and electron saturation regions are displayed on two separate linear scales, since the variations in the ion saturation regions are very small.

The behavior of the response in the electron retardation region (near $V=0$ Volts) is also as predicted by Langmuir cylindrical probe theory, as can be seen on figure 6. The expression used for comparison here was (Brace, 1998):

$$
I_{e}=2 \pi n_{0} r_{0} e \sqrt{\frac{e T_{e}}{2 \pi m_{e}}} \exp \frac{V}{T_{e}}
$$

This initial validation of the proposed algorithm confirms that the important physical mechanisms involved are being accurately represented in the model.

Additionally, further qualitative confirmation of the validity of the model is provided by figures 7 and 8 , which depict the consistent solution that was obtained for the potential distribution and the ion and electron density distributions. The figures shown here correspond to the simulation parameters mentioned previously, with an applied potential of 21 volts. Note that the $x$ and $y$ axes are displayed on a logarithmic scale to allow proper viewing of the important features, which occur mostly near the tether itself.

\section{PRESENT STATUS AND CONCLUSIONS}

A novel approach based on kinetic theory was presented for the 2-D modeling of the tether problem. The proposed algorithm was validated with the well-documented Langmuir cylindrical probe theory by simulating a 1-D problem. As mentioned in (Laframboise, 1966), one of the important difficulties in finding self-consistent solutions for such problems is finding an appropriate algorithm that will yield convergence in order to find the fixed point of the VlasovPoisson operator. To address this issue, a numerical algorithm for computing the Jacobian matrix of the Vlasov-Poisson operator was developed which allowed use of the Newton root finding method. This has dramatically increased the rate of convergence for the cases that were tested and shown here. Further improvements to the iterative root-finding process as well as the Vlasov-Poisson operator are needed in order to allow similar convergence for cases including a nonzero drift velocity in the background plasma. Future developments include the accurate modeling of tethers of arbitrary cross-sections, as well as magnetic field and time variation effects. 


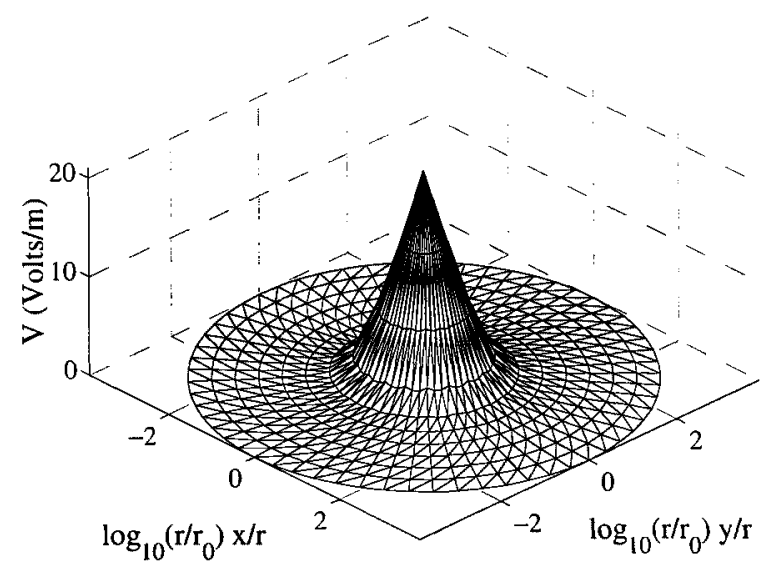

(a) Potential Distribution

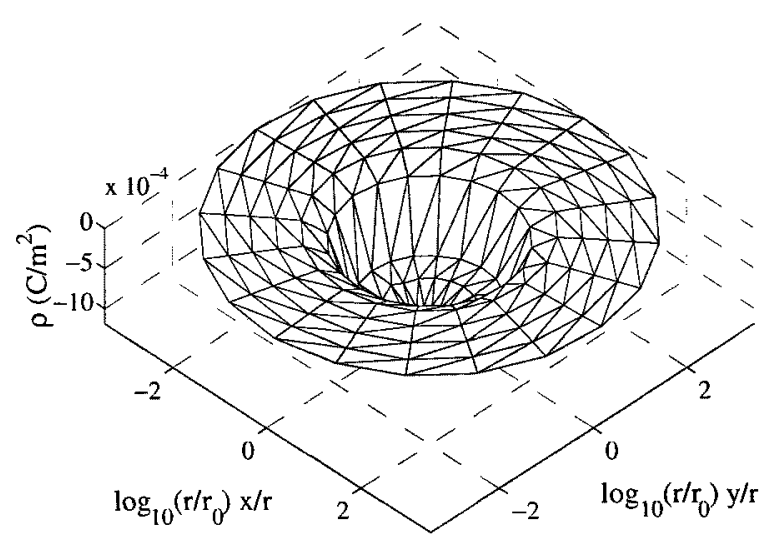

(b) Charge Density Distribution

FIGURE 7. Potential and Charge Density Distributions Around the Probe used in figure 6 (biased at $V_{p}=21 V$ ).

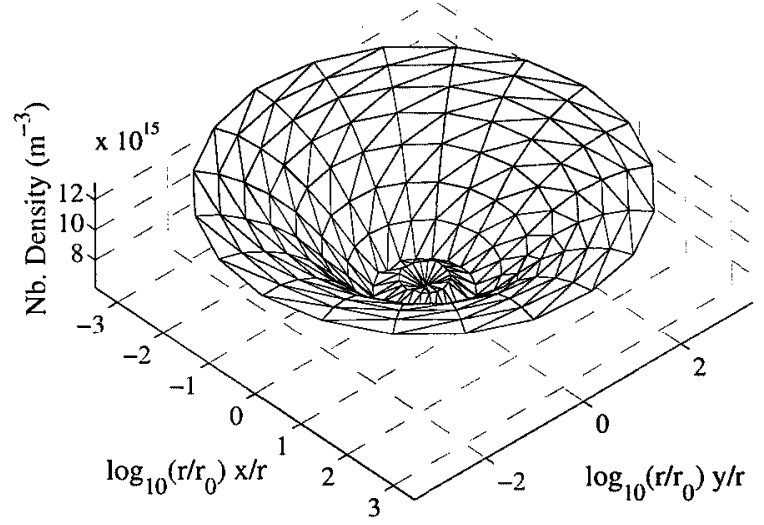

(a) Electron Number Density Distribution

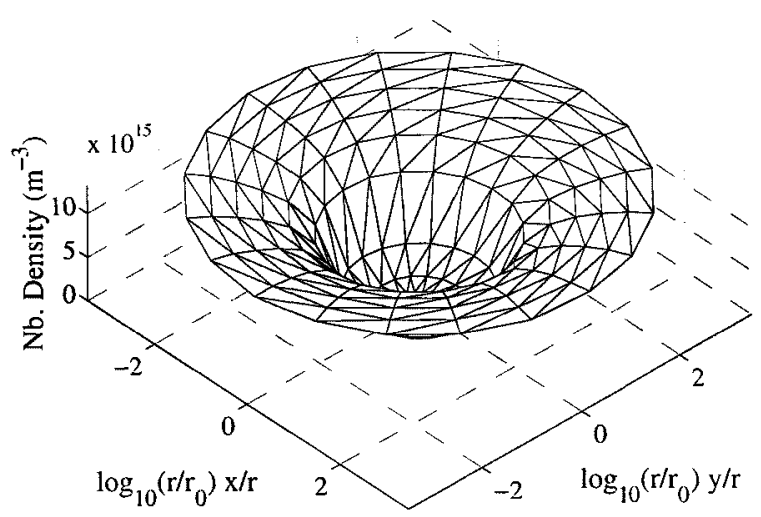

(b) Ion Number Density Distribution

FIGURE 8. Electron and Ion Number Density Distributions Around the Probe used in figure 6 (biased at $V_{p}=21 \mathrm{~V}$ ).

\section{NOMENCLATURE}

$\Delta v \quad$ Range of velocities accounted for on the outer boundary $(m / s)$

$E_{x} \quad x$ component of the electric field $(V / m)$

$E_{y} \quad y$ component of the electric field $(V / m)$

$f_{e} \quad$ Electron velocity distribution

$f_{i} \quad$ Ion velocity distribution

$\vec{g}(\cdot) \quad$ Fixed-point nonlinear operator composed of a combination of the Vlasov and Poisson solvers (input in Volts, output in Volts)

$\vec{h}(\cdot) \quad$ Fixed-point nonlinear operator based on the Newton method

$I_{\text {probe }} \quad$ Net collection current to the probe $(A)$
$I_{\mathrm{omI}}$

$I_{\mathrm{e}}$

$\mathbf{J}_{\mathbf{g}}$

$K_{\max }$

$m_{e}$

$m_{i}$

$n_{0}$

OML

$q_{e}, e$

$q_{i}$

$r_{0}$
OML theory-predicted collection current $(A)$ Electron current to the probe $(A)$ Jacobian matrix of operator $\vec{g}(\cdot)$. Range of kinetic energies accounted for on the outer boundary $(\mathrm{eV})$ Electron mass $(\mathrm{kg})$ Ion mass $(\mathrm{kg})$ Background plasma density $\left(m^{-3}\right)$ Orbital Motion Limit Electron charge $=+1.6021 \times 10^{-19} \mathrm{C}$ Ion charge $(C)$ Probe radius $(m)$ 
$r \quad$ Radial distance measured from the center of the tether conductor $(m)$

$r_{b c} \quad$ Radius of outer equipotential shell $(m)$

$\rho(x, y) \quad$ Net charge density distribution $\left(C / m^{2}\right)$

$\rho_{s}(x, y) \quad$ Surface charge density distribution $(C / m)$

$\vec{\rho} \quad$ Vector containing samples of the charge density distribution at mesh nodes $\left(\mathrm{C} / \mathrm{m}^{2}\right)$

$T_{e} \quad$ Electron temperature $(\mathrm{eV})$

$T_{i} \quad$ Ion temperature $(\mathrm{eV})$

$v_{x} \quad x$ component of velocity $(\mathrm{m} / \mathrm{s})$

$v_{y} \quad y$ component of velocity $(\mathrm{m} / \mathrm{s})$

$v_{d} \quad x$-directed plasma drift velocity $(\mathrm{m} / \mathrm{s})$

$V_{p} \quad$ Probe voltage $(V)$

$v_{\min , \max }$ Minimum/maximum velocity accounted for at any given position $(m / s)$

$V(x, y) \quad$ Electric potential distribution $(V)$

$V_{\text {plasma }}$ Plasma contribution to the electric potential distribution $(V)$

$V_{\text {obsc }} \quad$ Outer boundary surface charge contribution to the electric potential distribution $(V)$ Probe surface charge contribution to the electric potential distribution $(V)$ Vector containing samples of the potential distribution at the specified nodes $(V)$

\section{ACKNOWLEDGMENTS}

É.C. acknowledges the scholarship support of the Natural Sciences and Engineering Research Council of Canada as well as the Communications Research Centre Canada.

\section{REFERENCES}

Brace, L. H. "Langmuir probe measurements in the ionosphere," Measurement Techniques in Space Plasmas: Particles, Geophysical Monograph, pp. 23-34 (1998).

Cheng, D. K. Field and wave electromagnetics. Addison-Wesley Pub. Co., 1989.

Laframboise, J. Theory of Spherical and Cylindrical Langmuir Probes in a Collisionless, Maxwellian Plasma at Rest. Ph.D. thesis, University of Toronto, 1966.

Sanmartín, J. and Estes, R. "The orbital-motion-limited regime of cylindrical Langmuir probes," Physics of Plasmas 6, pp. 395-405 (1999).

Sikorski, K. A. Optimal Solution of Nonlinear Equations, Oxford University Press, 2001.

Stutzman, W. L. and Thiele, G. A., Antennna Theory and Design, John Wiley \& Sons, 1981. 